\title{
On the Capacity of Wireless Erasure Networks
}

\author{
Radhika Gowaikar, Amir F. Dana, Ravi Palanki, Babak Hassibi and Michelle Effros \\ Dept. of Electrical Engineering, Caltech \\ Pasadena, CA \\ \{gowaikar, amirf, palanki, hassibi, effros\}@caltech.edu
}

\begin{abstract}
We determine the capacity of a certain class of wireless erasure relay networks. We first find a suitable definition for the "cut-capacity" of erasure networks with broadcast at transmission and no interference at reception. With this definition, a max-flow min-cut capacity result holds for the capacity of these networks.
\end{abstract}

\section{INTRODUCTION}

For wireline networks, the max-flow min-cut result gives the network capacity between a single source and a single destination as well as in some multicast scenarios [1]. In this paper we consider a certain class of erasure wireless networks and show a max-flow min-cut capacity result for it. A detailed proof as well as extensions to some multicast scenarios can be found in $[2]$.

\section{MODEL}

We model the network by a directed acyclic graph $G=(V, E)$. Each edge $\left(v_{i}, v_{j}\right) \in E$ represents a memoryless erasure channel from $v_{i}$ to $v_{j}$ with erasure probability $\epsilon_{i, j}$ associated with it. All channels are assumed independent and operate without delay.

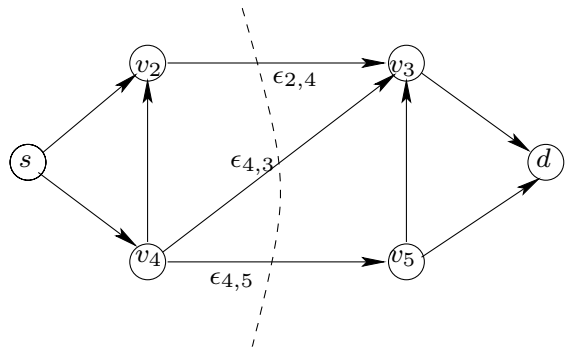

Figure 1: Example of a network. The capacity of the cut marked with a dotted line is $1-\epsilon_{2,4}+1-\epsilon_{4,3} \epsilon_{4,5}$

Let $s=v_{1} \in V$ be the source node that wishes to transmit a message to $d=v_{|V|}(\neq s) \in V$ which is the destination node. The other nodes simply have to aid this communication.

We incorporate broadcast in our network by insisting that vertex $v_{i}$ transmit the same symbol on all outbound edges. This implies that $v_{5}$ must transmit the same message on edges $\left(v_{5}, v_{3}\right)$ and $\left(v_{5}, v_{6}\right)$. For reception, we assume that $v_{i}$ receives the symbols from each incoming edge without interference. This means that $v_{2}$ receives messages from links $\left(v_{1}, v_{2}\right)$ and $\left(v_{4}, v_{2}\right)$ without their acting as interference for each other.

Finally, we assume that the decoder $d$ knows the exact erasure pattern that occurred on each link of the network. This

\footnotetext{
${ }^{1}$ This work was supported in part by the National Science Foundation under grant no. CCR-0133818, by the office of Naval Research under grant no. N00014-02-1-0578, and by Caltech's Lee Center for Advanced Networking.
}

assumption requires a serious overhead of data transmission for a regular bit erasure channel. However, if we assume that the links are packet erasure channels, this assumption is reasonable for very long packets.

\section{Definitions}

An $s-d$ cut is defined as a partition of the vertex set $V$ into two subsets $V_{s}$ and $V_{d}=V-V_{s}$ such that $s \in V_{s}$ and $d \in V_{d}$. Clearly, an $s-d$ cut is determined simply by $V_{s}$. For the $s-d$ cut given by $V_{s}$, let the cutset $E\left(V_{s}\right)$ be the set of edges defined below

$$
E\left(V_{s}\right)=\left\{\left(v_{i}, v_{j}\right) \mid\left(v_{i}, v_{j}\right) \in E, v_{i} \in V_{s}, v_{j} \in V_{d}\right\}
$$

Denote by $W\left(V_{s}\right)$ the value of an $s-d$ cut given by $V_{s}$. We define $W\left(V_{s}\right)$ below.

$$
W\left(V_{s}\right)=\sum_{i:\left(v_{i}, v_{j}\right) \in E\left(V_{s}\right)}\left(1-\prod_{j:\left(v_{i}, v_{j}\right) \in E\left(V_{s}\right)} \epsilon_{i, j}\right)
$$

Consider this definition of the value of the cut or the "cutcapacity". In the wireline case, the value of the cut is simply the sum of the capacities of each edge in $E\left(V_{s}\right)$. Since our system model incorporates broadcast the cut-capacity is the sum of the capacities of each broadcast system that operates across the cut. This gives the outer summation in the definition of $W\left(V_{s}\right)$ above. For the capacity of each broadcast system that operates across the cut, assume that the receiver nodes within that system get to co-operate and hence have an effective erasure probability $\epsilon_{\text {eff }}$ equal to the product term in (1). Hence the capacity of each broadcast system is $\left(1-\epsilon_{\mathrm{eff}}\right)$ which gives the quantity inside the summation.

In Fig. (1), the cut given by $V_{s}=\left\{s, v_{2}, v_{4}\right\}$ is marked with a dotted line. We have $E\left(V_{s}\right)=\left\{\left(v_{2}, v_{3}\right),\left(v_{4}, v_{3}\right),\left(v_{4}, v_{5}\right)\right\}$. This consists of the broadcast system emanating from $v_{4}$, viz., $\left\{\left(v_{4}, v_{3}\right),\left(v_{4}, v_{5}\right)\right\}$ and the (degenerate) broadcast system emanating from $v_{2}$, viz., $\left\{\left(v_{2}, v_{3}\right)\right\}$. The capacity of the former is $1-\epsilon_{4,3} \epsilon_{4,5}$ and that of the latter is $1-\epsilon_{2,4}$. Hence $W\left(V_{s}\right)=1-\epsilon_{4,3} \epsilon_{4,5}+1-\epsilon_{2,4}$.

\section{Main Result}

We can then prove the following max-flow min-cut theorem.

Theorem 1. The capacity of the erasure relay network described above is given by the value of the cut with minimum value.

$$
C=\min _{V_{s}} W\left(V_{s}\right)
$$

where $V_{s}$ determines an $s-d$ cut.

\section{REFERENCES}

[1] R. Ahlswede, N. Cai, S.-Y. R. Li, R. W. Yeung, "Network information flow," IEEE Trans. Info. Theory, vol. 46, pp. 12041216, 2000.

[2] A. F. Dana, R. Gowaikar, R. Palanki, B. Hassibi, M. Effros, "On the Capacity of Wireless Erasure Broadcast Networks," to be submitted to IEEE Trans. Info. Theory 